\title{
Imagery and self-efficacy in the injury context
}

\author{
Wesch, N.; Callow, N.; Hall, C.; Pope, J.P.
}

\section{Psychology of Sport and Exercise}

\author{
DOI: \\ 10.1016/j.psychsport.2015.12.007 \\ Published: 01/05/2016 \\ Peer reviewed version \\ Cyswllt i'r cyhoeddiad / Link to publication
}

Dyfyniad o'r fersiwn a gyhoeddwyd / Citation for published version (APA):

Wesch, N., Callow, N., Hall, C., \& Pope, J. P. (2016). Imagery and self-efficacy in the injury context. Psychology of Sport and Exercise, 24, 72-81.

https://doi.org/10.1016/j.psychsport.2015.12.007

\footnotetext{
Hawliau Cyffredinol / General rights

Copyright and moral rights for the publications made accessible in the public portal are retained by the authors and/or other copyright owners and it is a condition of accessing publications that users recognise and abide by the legal requirements associated with these rights.

- Users may download and print one copy of any publication from the public portal for the purpose of private study or research.

- You may not further distribute the material or use it for any profit-making activity or commercial gain

- You may freely distribute the URL identifying the publication in the public portal ?
}

Take down policy

If you believe that this document breaches copyright please contact us providing details, and we will remove access to the work immediately and investigate your claim. 


\section{Accepted Manuscript}

Imagery and self-efficacy in the injury context

Natasha Wesch, Nichola Callow, Craig Hall, J. Paige Pope

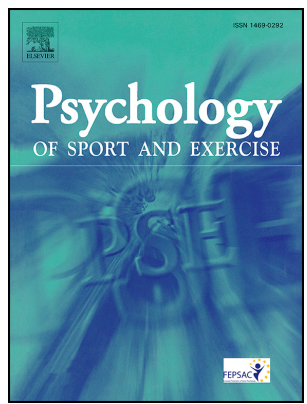

PII: S1469-0292(15)30034-0

DOI: $\quad$ 10.1016/j.psychsport.2015.12.007

Reference: PSYSPO 1061

To appear in: Psychology of Sport \& Exercise

Received Date: 14 November 2014

Revised Date: 23 December 2015

Accepted Date: 29 December 2015

Please cite this article as: Wesch, N., Callow, N., Hall, C., Pope, J.P., Imagery and self-efficacy in the injury context, Psychology of Sport \& Exercise (2016), doi: 10.1016/j.psychsport.2015.12.007.

This is a PDF file of an unedited manuscript that has been accepted for publication. As a service to our customers we are providing this early version of the manuscript. The manuscript will undergo copyediting, typesetting, and review of the resulting proof before it is published in its final form. Please note that during the production process errors may be discovered which could affect the content, and all legal disclaimers that apply to the journal pertain. 
RUNNING HEADER: Imagery in the injury context

TITLE: Imagery and self-efficacy in the injury context

Natasha Wesch

University of Western Ontario, Canada

nwesch@uwo.ca

Nichola Callow ${ }^{1}$

Bangor University, UK

n.callow@bangor.ac.uk

Craig Hall

University of Western Ontario, Canada

chall@western.ca

J. Paige Pope

University of Ottawa

j.paige.pope@gmail.com

${ }^{1}$ Corresponding author

Acknowledgement: The authors express their thanks to Robert Litchfield and Lorie Forwell from the Fowler Kennedy Sport Medicine Clinic, University of Western Ontario. Without them this research would not have been possible. Thanks are also extended to Lynne Evans for her advice on the adherence measure. 
Imagery and self-efficacy in the injury context

Injuries play a pivotal role in the careers of many athletes by causing both physical and psychological harm. How athletes manage their injuries can have a sizeable impact on their psychological and rehabilitation outcomes. Successful athletic injury rehabilitation is enhanced through proper adherence to a prescribed rehabilitation program (Bassett, 2006; Brewer et al., 2000). Further, previous research has shown that higher levels of self-efficacy are significantly associated with better adherence to such a program (Brewer et al., 2003; Milne, Hall, \& Forwell, 2005; Woodgate, Brawley, \& Weston, 2005). Therefore, self-efficacy may play an important role in an injured athlete's motivation to recover, subsequent adherence to a prescribed rehabilitation program, and treatment outcome.

A variable related to self-efficacy in rehabilitation is an injured athlete's imagery use (Milne et al., 2005; Wesch, et al., 2011). Imagery has been described as "an experience that mimics real experience, and involves using a combination of different sensory modalities in the absence of actual perception" (Cumming \& Ramsey, 2008, p.5). Injured athletes report using four types of imagery (Driediger, Hall, \& Callow, 2006; Rossman, 2002; Sordoni, Hall, \& Forwell, 2002). Cognitive imagery is used to rehearse rehabilitation exercises, and motivational imagery is used to set goals, control arousal levels and increase self-confidence. Healing imagery entails imagining the physiological processes taking place during rehabilitation (e.g., tissue and/or bone healing), whereas pain management imagery involves images of pain dissipating or images that can help the athlete cope with the pain associated with an injury.

Milne et al. (2005) investigated the relationships between injured athletes' use of cognitive, motivational, and healing imagery and both task efficacy (confidence in one's ability to perform the fundamental aspects of a task in a specific situational context) and coping efficacy 
(confidence in one's ability to perform a task under challenging conditions or to overcome social, personal and environmental constraints). Imagery was assessed using the Athletic Injury Imagery Questionnaire (AIIQ-2; Sordoni et al., 2002) and self-efficacy was measured with the Athletic Injury Self-Efficacy Questionnaire (AISEQ; Milne et al., 2005; Sordoni et al. 2002). The questionnaires were administered in an outpatient physiotherapy clinic to 270 injured athletes, and cognitive imagery was found to be significantly associated with task efficacy but no other significant relationships emerged.

A limitation of the AIIQ-2 and consequently research that has employed it (e.g., Milne et al. 2005) is that cognitive, motivational and healing imagery are assessed but not pain management imagery. Pain is the most pervasive and debilitating obstacle to effective rehabilitation experienced by injured athletes. It has significant physical and psychological effects in almost every aspect of recovery (Arvinen-Barrow \& Walker, 2013; Heil, 1993). Therefore, pain management imagery may be an important resource for athletes involved in injury rehabilitation. Further, qualitative analysis does highlight that injured athletes use this type of imagery (e.g., Driediger et al., 2006). Thus, pain management imagery needs to be measured in order to overcome the limitation of the AIIQ-2 and previous injury-related imagery research that has used it. Consequently, in Study 1, the AIIQ-2 was adapted to include a pain management imagery subscale and the factorial validity tested. The aim of Study 1 was to develop a measure reflecting a broader range of types of imagery that injured athletes report using, which could then be used in Study 2.

Another limitation of the imagery rehabilitation research is the lack of intervention studies conducted in this context. Given injured athletes report using imagery extensively during rehabilitation (Driediger et al., 2006; Evan, Hare, \& Mullen, 2006) and that imagery 
interventions have proven to be effective in both sport and exercise (e.g., Duncan, Rodgers, Hall, \& Wilson, 2011; Munroe-Chandler, Hall, Fishburne, Murphy, \& Hall, 2012), it is somewhat surprising that only a limited number of imagery interventions have been conducted in the injury rehabilitation context. Strong support for an imagery intervention in injury rehabilitation is provided from a randomized-controlled trial (Cupal \& Brewer, 2001), which examined the effect of guided imagery and relaxation on knee strength, re-injury anxiety, and pain among patients undergoing surgical reconstruction of the anterior cruciate ligament reconstruction. Results demonstrated significantly greater knee strength and significantly less re-injury anxiety and pain at 24-week post-surgery among the intervention compared with the placebo and control conditions.

The only imagery intervention study considering self-efficacy was recently conducted by Maddison et al. (2011). The aim of this study was to evaluate the effectiveness of a guided imagery and relaxation program on improving functional outcomes post-anterior cruciate ligament repair delivered over nine individual sessions as an adjuvant treatment to standard rehabilitation. A randomized controlled trial was conducted. Participants were randomized to guided imagery and standard rehabilitation or standard rehabilitation alone (control). The primary outcome was knee strength 6-month post-operatively, but other variables were also examined including knee laxity and self-efficacy. Following the intervention, the groups did not differ on knee strength but the intervention group demonstrated a significant positive effect for knee laxity. In addition, there was a significant group by time interaction for self-efficacy. Although self-efficacy decreased over time for both groups, in the intervention group it remained fairly stable between weeks 6 and 12 compared with the control group. However, the study by Maddison et al. (2011) has several limitation worthy of identification. Specifically, the 
intervention combined imagery and relaxation, thus it is not possible to partial out the independent influence of imagery on the outcome variables. In addition, a composite score for task and coping efficacy was used rather than examining each separately. Despite the limitations of this study, theoretically it is understandable that imagery may maintain or even enhance selfefficacy. Indeed, Bandura (1997) states that self-efficacy beliefs are constructed from four principle antecedents: enactive mastery experience, vicarious experience, verbal persuasion, and physiological and affective states, with imagery increasing self-efficacy via these antecedents. Related to the present context, for example, by using cognitive imagery (e.g., prior to performing a rehabilitation exercise, I am able to image myself completing it perfectly) injured athletes could gain enactive mastery experiences which may increase task efficacy.

Given the paucity of intervention research in the injury context, and that research highlights changes in imagery use across early, mid and late phases of injury rehabilitation (Hare, Evans, \& Callow, 2008) it would be prudent to conduct more research in this area, and to focus on one phase of rehabilitation. Further, the research investigating imagery use and selfefficacy have done so while the patients have been undergoing physiotherapy, rather than prior to commencing treatment, yet self-efficacy is proposed to be important for patients about to commence a rehabilitation program (cf. Lox, Martin Ginnis, \& Petruzzello, 2014). Thus, using a multiple-baseline single-subject design, Study 2 applies an imagery intervention in an attempt to improve the psychological state of five patients about to commence physiotherapy treatment through the use of imagery and its effects on task and coping efficacy. Based on the theoretical rationale that imagery enhances self-efficacy via its antecedents, it was hypothesised that the imagery intervention would result in higher task and coping efficacy in comparison to baseline.

\section{Study 1}




\section{Method}

Participants. A sample of 292 injured athletes were recruited from a Canadian University Sport Medicine Clinic. Seven participants were excluded due to missing or incomplete data. The final sample consisted of 285 sport rehabilitation participants, $47 \%$ men $(n=134)$ and 53\% women $(n=151)$, aged 18 to 74 years $(M=28.64, S D=14.30)$, who had sustained diverse injuries. Inclusion criteria were that participants had to be 18 years of age or older and engaged in physiotherapy for at least one week. Participants were from 36 different sports, with ice-hockey reported most frequently $(9.10 \%)$ followed by alpine skiing $(8.80 \%)$ and soccer $(8.10 \%)$. Level of participation was reported as either recreational $(n=149)$, provincial $(n=49)$, varsity $(n=55)$ or national $(n=39)$.

\section{Measures}

Athletic Injury Imagery Questionnaire (AIIQ-2; Sordoni et al., 2002). The AIIQ-2 consists of twelve items delineating the three types of imagery: cognitive imagery "Before performing a rehabilitation exercise, I imagine myself completing it perfectly", motivational imagery "I imagine myself achieving my treatment goals", and; healing imagery "I imagine my body repairing itself." Participants rate their imagery use on a 9-point Likert scale from 1 (never) to 9 (always), indicating their use of that particular type of imagery. Previous research (Sordoni et al., 2002; Milne et al., 2005) has found the AIIQ-2 to be both valid and reliable for the subscales measured.

\section{Adaptation of the AIIQ-2 (AIIQ-3)}

Based on theoretical and applied imagery research, three imagery experts developed four pain management items (e.g., "To distract myself from the pain associated with my injury, I use imagery"). In line with the items comprising the other three subscales, the four pain management 
items reflected a combination of content and function (for a discussion of the imagery content and function debate see Cumming \& Williams, 2012). These items formed a pain management subscale, and were added to the AIIQ-2 to create the AIIQ-3, thus resulting in a 16-item measure. A substantive validity test was conducted to assess if the items were judged to be reflective of, or theoretically linked to, the constructs under study (Holden \& Jackson, 1979). This test was achieved by five research experts and ten injured athletes conducting an item sort task of the 16 items from the AIIQ-3. All respondents sorted the 16-items into the corresponding imagery function, thus indicating $100 \%$ accuracy in the sorting task, or high substantive validity (Anderson \& Gerbing, 1991).

\section{Procedure}

The study was approved by the university's ethics committee. Injured athletes were approached immediately following their physiotherapy session at the University Sport Medicine Clinic. They were given a letter of information and informed consent form, if participants agreed to participate and signed the form they were administered the AIIQ-3. All participants who were approached volunteered for the study.

\section{Data Analysis and Results}

Descriptive results of the AIIQ-3 are presented in Table 1. The factorial validity of the AIIQ-3 was tested using AMOS 23 with maximum likelihood estimation procedures. Mardia's coefficient score (Multivariate Kurtosis $=64.99$; critical ratio $=22.86$ ) indicated that the sample data deviated from multivariate normality. Therefore Maximum Likelihood (ML) with bootstrapping (1000 bootstrapped samples with replacement from the original sample; Preacher \& Hayes, 2008) was conducted as ML is recommended when the normality assumption is violated (West, Finch, \& Curran, 1995). The following results were revealed: $\chi^{2} 2=339.50, \mathrm{df}=$ 
$98, \chi^{2} 2 / \mathrm{df}=3.46, \mathrm{CFI}=.92, \mathrm{ILI}=.90, \mathrm{RMSEA}=.09(90 \% \mathrm{CI}=.08-.10) \mathrm{SRMR}=-.06$. The bootstrap-generated factor loadings and standard errors are reported in Table 2, and highlight that each item contributed meaningfully to its respective scale. The chi-square statistic was significant, suggesting that the model was not of adequate fit to the data and the $\chi^{2} 2 / \mathrm{df}$ ratio was above the 2.0 cut-off; however, chi-square is sensitive to large sample sizes (greater than 200) and significant results are often found in empirical research (Hayduck, 1996). Therefore, other fit indices were considered as measures of model fit. The ILI and CFI both met the recommended criteria of .90 or higher. The RMSEA was slightly above while the SRMR was below the criteria of less than or equal to .08 which is considered adequate fit. Taken together, the results indicate that the model was an adequate fit to the data.

\section{Discussion}

Previous research has demonstrated that self-efficacy plays an important role in an injured athlete's motivation to recover, subsequent adherence to a prescribed rehabilitation program, and treatment outcome (e.g., Woodgate et al., 2005). A variable related to self-efficacy in rehabilitation is an injured athlete's imagery use (Milne et al., 2005; Wesch, et al., 2011). However previous imagery and injury research has been limited because the main imagery measurement tool the AIIQ-2 does not measure pain management imagery, which is an important type of imagery. Consequently, Study 1 set out to rectify this, by adapting the AIIQ-2 to include a pain management subscale.

Results supported a four factor structure for the AIIQ-3 consisting of cognitive, motivational, healing, and pain management imagery. Thus, a measure reflecting a broader range of imagery types reported by injured athletes that demonstrated initial support for factorial validity resulted. It is recommended that researchers conduct further psychometric testing of the 
questionnaire (e.g., comparing competing factor structures), and given further supportive findings, use this version of the AIIQ in future injury imagery research in which the AIIQ scores are a primary dependent variable. The results of Study 1 , however, provide adequate evidence for employing the AIIQ-3 in Study 2 as a manipulation check (not a primary outcome variable). Specifically, the purpose of Study 2 was to explore the effect of an imagery intervention on selfefficacy, and the AIIQ-3 was used to establish if the imagery intervention increased imagery use. Further, the intervention content in Study 2 was guided by the results of Study 1 in that all four types of imagery were administered.

\section{Study 2}

\section{Method}

Participants. Six participants, two men and four women, aged 18 to 65 years $(M=49.50$, $S D=16.56$ ), were recruited for this study. Participation in recreational walking was the physical activity of choice reported most frequently $(n=4)$, followed by rugby $(n=1)$, and horseback riding $(n=1)$. Level of participation in this physical activity was reported as either recreational $(n=5)$ or competitive (i.e., provincial) $(n=1)$. Inclusion criteria were that participants had to be 18 years of age or older, to have sustained a Type B malleolar fracture treated operatively within 72 hours prior to the initial contact, to be immobilised and non-weight bearing for 6 weeks following surgery, and to be able to comply with the weekly demands of the intervention. Participants were not from Study 1.

\section{Measures}


Athletic Injury Imagery Questionnaire (AIIQ-3). The AIIQ-3 developed in Study 1, which reflects the broader range of imagery types that injured athletes report using, was administered to the participants. ${ }^{1}$

Athletic Injury Self-Efficacy Questionnaire (AISEQ; Milne et al. 2005). Self-efficacy was evaluated using the AISEQ, which is comprised of 7 items representing two types of selfefficacy, task and coping. An example task item is "I am confident that I can perform all of the required rehabilitation exercises". An example coping efficacy item is "I imagine coping with the pain associated with my injury.” The participants rate their self-efficacy on a $100 \%$ confidence scale ranging from $0 \%$ (no confidence) to $100 \%$ (completely confident). The AISEQ has sound psychometric properties; $\chi^{2} / d f=1.82, \mathrm{AGFI}=.94, \mathrm{CFI}=.98$, and RMSEA $=.06$ (Milne et al., 2005).

Social validation. A social validation procedure was used to determine the participants' satisfaction with the intervention in terms of effectiveness (Page \& Thelwell, 2013). Specifically, through the use of a log book participants were asked to provide a general description of the content of their imagery session and then to rate the effectiveness of the imagery session on a 5point Likert scale ( $1=$ not at all effective; $5=$ highly effective). Only participants receiving the intervention were asked to complete the imagery log. Further, through the use of a postexperimental interview, participants' views on the intervention were elicited in order to establish both the satisfaction (or not) of the intervention, and to aid interpretations of any intervention effects (See Appendix A for interview questions administered to the intervention participants) ${ }^{2}$.

\section{Procedure}

\footnotetext{
${ }^{1}$ The AIIQ-3 is available on request from the third author.

2 The interview questions administered to participant 6 are available on request from the first author.
} 
The study was approved by the university's ethics committee. Patients who fit the inclusion criteria were approached by the referring orthopaedic surgeon and asked if they would be interested in participating in a study aimed at helping them improve their psychological readiness for physiotherapy treatment. A standard protocol for Type B malleolar fracture surgery was in place, that is after surgery and 6 weeks of non weight bearing, physiotherapy commences. Thus, the lead author contacted those willing to take part in the study within 72 hours of surgery, with the first meeting date arranged within one week post-surgery. For this first meeting only, the majority of participants were met at their home due to mobility issues following lower limb surgery. At this time, participants received a letter of information, were given a description of the study, and were asked to sign a consent form and to complete two questionnaires to measure participants' imagery use and self-efficacy. These questionnaires were then completed 11 times over the course of the six-week period between the first meeting and the commencement of physiotherapy treatment. Participants also kept an imagery log. Because we were restricted by the number of weeks for the baseline period (i.e., six weeks) rather than administer the intervention when the baseline had stabilised (cf., Kazdin, 2010) the start of the imagery intervention was randomly assigned to 5 out of the 6 participants at week 2 , week 3 , week 4 , and week 5. One participant did not receive the imagery intervention, rather he/she just received the general malleolar fracture surgery and rehabilitation information (see the next section for more detail on the intervention and information). Biweekly calls were made by a researcher to remind the participants to complete the questionnaires and to answer any questions. Three weeks after commencement of physiotherapy, each participant received the post-experimental interview.

Intervention. The intervention was administered by the first author of the present study. Strict instructions for the intervention were written down, scrutinized to avoid possible 
occurrences of experimenter bias, and were adhered to throughout the intervention ${ }^{3}$. The intervention took place in two sessions (each approximately 45-60 minutes in length). The sessions were held one week apart to enable participants to better retain the information provided in each meeting and to limit the amount of consecutive daily travel required by each participant. In the first intervention session participants were introduced to the anatomy of the ankle joint and surrounding structures through the use of an anatomical model and wall chart. Participants also were provided with an overview of the surgery protocol for a Type B malleolar fracture including an example of an X-ray of an ankle pre and post surgery. Participants then were introduced to imagery use in injury rehabilitation (i.e., cognitive, motivational, healing and pain management imagery). Finally, scripts for healing and pain management imagery were introduced. At the end of this session, participants were asked to practice their imagery daily and were given an imagery log in which to record their imagery use.

The second intervention session commenced with an opportunity to answer questions, followed by the introduction of the cognitive and motivational imagery scripts. Following the completion of the scripts, the imagery log use was reviewed. At the end of this session participants were provided with an outline of 10 injury-specific rehabilitation exercises one may encounter in the first few weeks of physiotherapy, and a CD containing voice files of the scripts for all four injury rehabilitation imagery functions. Participants were encouraged to use the outline of rehabilitation exercises and the CD to help them during their imagery sessions and were reminded to practice their imagery daily and record their imagery use. Participants were not provided with specific details as to which type of imagery use to practice, but were encouraged to use the type of imagery that best suited their needs at the time.

\footnotetext{
${ }^{3}$ The instructions are available on request from the first author.
} 
Malleolar fracture surgery and rehabilitation information scripts. The information scripts, which contained general information about the surgery protocol and expected physiotherapy treatment protocol, were created with the assistance of the referring surgeon and the head physiotherapist at a major sport medicine clinic. Participants were provided with an overview of the anatomy of the ankle joint and surrounding structures, the surgery protocol for their specific injury, what could be expected in terms of pain and discomfort, and what they could expect in the first few weeks of physiotherapy in terms of protocol. ${ }^{4}$

Imagery scripts. Imagery scripts were created around the four types of imagery use in injury rehabilitation (healing, pain management, cognitive and motivational) and were written with both response and stimulus propositions but with an emphasis placed on response propositions (cf., Lang, Kozak, Miller, Levin, \& McLean, 1980). See Appendix B for extracts from the respective imagery scripts. ${ }^{5}$

\section{Data Analysis}

Manipulation check. In order establish that the imagery intervention resulted in imagery usage by athletes, descriptive analyses (mean and standard deviation) were conducted on the baseline and post-intervention imagery use data as assessed by the AIIQ-3.

Intervention effects. Visual analysis was used to examine possible treatment effects of the imagery intervention on self-efficacy across the baseline and experimental condition (Kazdin, 2010). Specifically, task and coping efficacy data for within and across pre-intervention (baseline) and post-intervention (experimental) phases for each participant were analyzed through five key features of the graphically displayed data: (a) level, (b) variability, (c) trend, (d) immediacy of effect, and (e) overlap (Kratcohwill et al., 2010). Level refers to the approximate

\footnotetext{
${ }^{4}$ Information scripts are available on request from the first author.

${ }^{5}$ The imagery scripts for all four types are available on request from the first author.
} 
mean within each phase and variability to the approximate deviation within each phase. Trend refers to the approximate slope of the phases, and, in the present study, immediacy of effect to the change in level from the last data point in pre-intervention to the first three data points postintervention. Further, descriptive and statistical analyses were used to compliment visual inspection (Kinugasa, 2013). That is, mean and standard deviation self-efficacy scores for each phase, percentage of non-overlapping points (PND; Scruggs \& Mastropieri, 1998), and Standardized Mean Difference (SMD all; Rosnow \& Rosenthal, 1996) were calculated.

In line with Martin and Pear (1996), interpretation of the results with an indication of positive effect centred on: (a) the baseline performance being in a stable or opposite direction to that of the predicted effects of the intervention; (b) the greater number of times that an effect was replicated both within and across participants; (c) a fewer number of overlapping data points between baseline and treatment, with a PND of $90 \%$ representing very effective treatment, $70 \%$ $89 \%$ representing effective treatment and $50 \%$ or below being ineffective (Scruggs \& Mastropieri, 2001); and (d) a $\mathrm{SMD}_{\text {all }}$ of .25 indicating a large effect size and .09 indicating a medium effect size (Cohen, 1988). However, it must be noted that defining single subject design effect size magnitudes in comparison to Cohen's specifications is open to debate, and should be interpreted with caution (Gage \& Lewis, 2013).

Post-experiment interview. The interviews were transcribed verbatim (producing 17 pages of single lined text), and for the purpose of the present study the answers to the following three questions were examined in detail: 1 . please discuss the usefulness of the intervention in preparing you for your physiotherapy treatment, 4. do you feel the imagery sessions were useful above and beyond the general information provided to you? Why? and, 9. please provide a general statement on the overall effectiveness of the intervention. Specifically, in order to 
provide evidence for the effectiveness (or not) of the intervention, and to aid interpretations of any intervention effects (see Page \& Thelwell, 2013 for a discussion of social validation), the transcribed interviews were read by each of the authors and a consensus reach on each of the raw quotes to be included in the results section.

\section{Results}

\section{Manipulation Check}

The mean imagery and standard deviation imagery results for the five intervention participants for baseline and post-intervention were $3.16(.82)$ and $5.35(.80)$ respectively, thus indicating that the intervention increased imagery usage. The mean score for the control participant was $4.23(.07)$.

\section{Intervention Effects, Self-efficacy}

The graphed task-efficacy and coping efficacy data are presented in Figures 1 and 2 respectively. Additionally, results for task-efficacy and coping efficacy level, variability, trend and immediacy of effect are presented in Tables 3 and 4 respectively.

Task-efficacy. Participant 1's data reveals a stable baseline trend and an intervention effect. The intervention effect is evidenced by a sizable level (mean) increase, an immediate effect following the intervention, and an increasing post-intervention phase trend in comparison to the baseline trend. Further, the PND was $100 \%$; however $\mathrm{SMD}_{\text {all }}$ was zero due to the zero standard deviation for the baseline data. Visual inspection of the data for Participant 2 did not demonstrate a change in trend from the baseline to the post-intervention phase. Similarly, the data did not present a treatment effect following the intervention. The PND was 50\% and the $\mathrm{SMD}_{\text {all }}$ was 1.44. Participant 3's data did not show a change in trend from the baseline to the intervention phase, nor was there a clear treatment effect following the intervention. The PND 
was $40 \%$, however $\mathrm{SMD}_{\text {all }}$ was zero due to the zero standard deviation for the baseline data. Visual inspection of the data for Participant 4 did not demonstrate a change in trend from the baseline to the post-intervention phase. Nevertheless, the data revealed an observable treatment effect immediately following the intervention evidence by a change in level, albeit small; and a reduction in variability at post-intervention. In addition, the PND was $100 \%$ and the $\mathrm{SMD}_{\text {all }}$ was 8.20. It must be noted that there may have been a ceiling effect with this participant's data. Participant 5's data illustrate a change in direction predicted for the effects of the intervention, as well as a clear treatment effect that occurred immediately following the intervention. The PND was $100 \%$ and the $\mathrm{SMD}_{\text {all }}$ was 3.07 .

Taken together, interpretation of the graphed data and statistical results indicates for two out of the five participants (participants 1 and 5) there were observable and statistically meaningful increases in task-efficacy due to the intervention. For Participant 4 there was a small treatment effect, with a possible ceiling effect evident. In contrast, for participants 2, 3 (who received the intervention) and Participant 6 (who did not receive the intervention) there was no change in task-efficacy over the six-week period between surgery and commencement of physiotherapy.

Coping efficacy. Visual inspection of the data for participant 1 reveals a stable baseline trend and an intervention effect. The intervention effect is evidenced by a sizable level (mean) increase, an immediate effect following the intervention, and an increasing post-intervention phase trend in comparison to the baseline trend. The PND was $100 \%$, however $\mathrm{SMD}_{\text {all }}$ was zero due to the zero standard deviation for the baseline data. Visual inspection of the data for participant 2 demonstrated a slight decreasing trend in coping efficacy during the baseline phase with stable trend following the intervention. However, these data coupled with the negligible 
level (mean) increase does not reveal a clear treatment effect, despite the PND of $100 \%$ and the $\mathrm{SMD}_{\text {all }}$ of 2.10. Visual inspection of participant 3's data did not demonstrate a change trend from the baseline phase to the post-intervention phase, and no visible treatment effect following the intervention. Indeed, the PND was $20 \%$ and the $\mathrm{SMD}_{\text {all }}$ was zero, again due to the zero standard deviation for the baseline data. Participant 4's data did not illustrate a change in trend from the baseline phase to the post-intervention phase, yet there was an observable treatment effect following the intervention indicated by the immediate change in level. In addition, the PND was $83 \%$ and the $\mathrm{SMD}_{\text {all }}$ was 3.16. Visual inspection of the data for participant 5 revealed an increasing trend for both baseline and intervention, but with a delayed treatment effect, the PND was $80 \%$ and the $\mathrm{SMD}_{\text {all }}$ was 1.88 .

Taken together, interpretation of the graphed data and statistical results indicates for three of the five participants (participant $1,4,5$ ) there were observable and statistically meaningful increases in coping efficacy due to the intervention. In contrast, for participants 2 and 3 (who received the intervention) and Participant 6 (who did not receive the intervention) there was no change in coping efficacy over the six-week period between surgery and commencement of physiotherapy.

Social validation. Participant 1 did not complete the imagery log book, however participants 2, 3, and 4 rated the imagery sessions as highly effective, and participant 5 rated the imagery sessions as moderately to highly effective.

Post-experimental interview. All intervention participants found the intervention to be very helpful or beneficial. For example, Participant 1 stated:

It was the fact that um, I was imagining myself, imagining you know like ah taking a piece of tape off, you know going, ffftt, like that. Or just do it slowly and take deep 
breaths at the same time and that's what I was doing in my mind when I was doing some of these exercises that, we were doing. And uh, that helped. I'd say that's the most beneficial thing right there because it prepared me mentally for what was going to be expected of me. And so when I came it was almost like a deja-vue. I knew, you know, that's what was happening.

Similarly, Participant 2 took a generic view of the beneficial effects of the intervention:

So but it's, it's [the imagery] great. It really, it really really is. I mean I'm a person who's mind over body anyway. It, but it is it's great, it's worked very well for me. Very well for me.

Whereas Participant 3 focused on a specific type of imagery:

Um, I thought it [the intervention] was very beneficial....and then the pain imagery was, ah, was useful prior to the physio and when I was healing, but also during physio when I was in pain, um it helped to eliminate that and move forward. Um, also the imagery of course of the physio exercises was useful because I did a lot of them, um, so I was prepared for that. Um, I used the pain imagery more frequently, um, several times a day in the beginning, ah, but at the same time I used the other ones quite a bit too just not, ah, as often I found.

The participants thought that the imagery sessions were useful and beyond the general information provided because the imagery worked, but also complimented the general information provided. For example, Participant 5 stated:

It really did, yes, it worked together, yes. I really appreciated the, the stuff because I had no idea. I think doctors, because they're so pressed for time now they don't really have time to explain to you what has happened to you. And I mean, uh, the first time I saw the X-ray, and I, I think I mentioned to you that there was not just one screw in the one area 
but in fact two, it was helpful to know. I like to know what's in my body [chuckle]. Um, so having a visual idea of what's happened to you, is, is very good....

All of the intervention participants thought that overall the intervention was very effective. Indeed Participant 1 stated:

It was a good fluke, it help me. At the end of the day, had I not taken part in this I don't think, because nobody else took the time to work with me on what I was gonna do. The physiotherapist even brought lots of, ok here, this you know, he didn't even show me, I actually had to ask for the x-ray but. By you showing me what it was and by you working, telling me the exercises, the pain and what it was gonna be, it allowed me in my mind, and that's the biggest thing, people have a fear, oh the pain's gonna be there or what the heck's going on with me? I'm glad that Bob introduced me to you because it really allowed me to work through, mentally, and prepare me mentally to do what I had to do to get myself back to where I'm at now. In fact, it allowed me to extend myself beyond what I, my capabilities would have been originally had I not done it [the intervention]. Because I was still in very ginger, saying ok I could have been, I'd say I'd be about three weeks behind where I am now, maybe a month. But because of our sessions and listening to the $\mathrm{CD}$, and doing those exercises that, and knowing the fact I'm breaking down scar tissue versus hurting the bone or the screws or anything like that. That pain was scar tissue, and it was a big help. And I can't, I have to attribute most of my recovery to you. You gave me the tools, but if you wouldn't have given me the tools I wouldn't have been able to do the work, so there you go. So yes, very pertinent, and yeah.

Whereas Participant 4 stated: 
Yeah, yeah well I just wanted to add um, ...Like if it wasn't for your sessions I don't think I would be as successful, because just, well there's just ah, a whhh, how do you recover? Like the surgeon doesn't tell you, he just says well it looks fine, you're on schedule. Ok, but what am I supposed to be doing [chuckles]? You know, sitting there at home wondering well, am I going to be able to walk again? You know, so this is tremendously helpful....Yeah so I mean, and that's, in that sense it's extremely valuable, for recovering from any broken limbs. You know, because it, there, you do, I mean the physiotherapist will help you but there's that huge amount of weeks that you're just waiting so that you can do physio, you know. What do you do? You know that [imagery] can be a positive influence.

Interestingly, Participant 6, who received the general information but not the imagery intervention, found the general information useful. Specifically, he/she stated:

Well it was very useful because I wasn't clear as to, I hadn't been shown any pictures at that point. When I was home, I, from my stay in the hospital, which was from the time it happened till I left, which was about 4 days, I hadn't been shown any pictures, I hadn't been shown anything as to what really had happened.....And um, even though I still don't understand all the proper terms of everything at least reading through everything and you going through it with me helped a lot because it made me understand that, what was really happening. And once the healing was done it was, the healing, the bones healed on schedule but then I had all this other work to do.

\section{Discussion}

Imagery research within athletic injury rehabilitation has received attention (e.g., Evans, et al., 2006; Milne et al., 2005); however, there has been no research aimed at improving the self- 
efficacy of patients about to commence physiotherapy treatment via an imagery intervention. The results here provided some support for the effectiveness of the imagery intervention. Specifically, interpretation of the graphed data and statistical results indicated that for two out of the five participants there were observable and statistically meaningful increases in task efficacy, and for another participant there was a small treatment effect, with a possible ceiling effect evident. For coping efficacy three of the five participants had observable and statistically meaningful increases due to the intervention. These findings are complemented by the results from the post-interview data where all intervention participants reported the intervention to be beneficial and effective, beyond the general information provided.

These findings support research by Maddison et al. (2011) showing that an imagery intervention can benefit self-efficacy during injury rehabilitation, and supports Bandura's (1997) view that imagery is an antecedent of self-efficacy. Overall, imagery use increased across the course of the intervention for all participants with the post-interview data indicating pain management and healing imagery were especially important in their recovery, with certain participants reporting that they changed the type of imagery they used during the time-course of the injury rehabilitation process (from pain management through to motivational imagery). The differential use of various types of imagery, and change in use across the various phases of injury rehabilitation has been reported in previous research (Evans et al., 2006; Hare et al., 2008).

For participants 2 and 3, no treatment effect was found for either type of self-efficacy. Nevertheless, like the other participants who received the intervention, they thought the intervention was helpful in preparing them for physiotherapy. Similar to the other patients, they also reported that the intervention reduced their anxiety and allowed them to play an active role 
in the healing process. These results are encouraging as previous medical research has shown that patients who are better prepared and informed prior to receiving interventions achieve better treatment adherence and therapeutic outcomes than standard care patients who do not receive a psycho-educational pre-treatment preparation session (e.g., Mahler \& Kulik, 2002).

Participant 6 did not receive the intervention, but received information pertaining to malleolar fracture surgery and rehabilitation treatment protocols (this information was also provided to participants who received the intervention). Interestingly, task and coping efficacy data demonstrated a very slight, yet noticeable increase, which occurred at the assessment immediately following the time point when the additional protocol information was given. As reported, the participant did state in the post-experimental interview that he/she found the general information useful. However, this rise in self-efficacy levels did not continue over the course of the study. It is possible that the protocol information influenced self-efficacy levels to a certain degree. Moreover, imagery use for this participant tended to decrease or remain stable, which is consistent with previous research (Wesch et al., 2011) that showed that overall imagery use remains relatively stable during rehabilitation if no imagery intervention is administered.

There is at least one limitation of the present study. Specifically, imagery ability was not measured. Previous research recommends that imagery ability is measured, with participants having at least a predetermined (moderate) level to ensure that the imagery intervention has the potential for an effect (see Roberts et al, 2008). However, we did not want to overload the participants with questionnaires at such a difficult time for them, and therefore decided not to administer an imagery ability questionnaire. Moreover, no ability measures exist for healing and pain management imagery. 
There are several practical implications that stem from the present findings. Providing patients, who have sustained a Type B malleolar fracture and been treated operatively, with an imagery invention is likely to produce some positive effects during their subsequent rehabilitation including enhance self-efficacy. Moreover, it is very likely these same benefits can be realized with other types of injuries where patients are required to wait some time before commencing physiotherapy.

\section{Conclusion}

Taking the results of the two studies together, we have provided an adapted version of the AIIQ-2 to overcome an inherent weakness in previous injury-related imagery research where pain management imagery has not been measured. Further, we have provided the first rehabilitation intervention based on the four types of imagery, with application of the AIIQ-3. Certainly, additional research is warranted to support the present findings but it seems quite clear that techniques for enhancing patients' preparedness, such as an imagery intervention, will have positive effects on rehabilitation. Future research should also take a longitudinal approach to examining adherence and post-surgery recovery in order to explore the possible beneficial effects of imagery on adherence and recovery during the full time course of rehabilitation and return to sport. 


\section{References}

Anderson, J. C. \& Gerbing, D. W. (1991). Predicting the performance of measures in a confirmatory factor analysis with a pretest assessment of their substantive validity. Journal of Applied Psychology, 76, 732-740.

Arvinen-Barrow, M. \& Walker, N. (2013). The psychology of sport injury and rehabilitation. Oxon, UK: Routledge.

Bandura, A. (1997). Self-efficacy: The exercise of control. NY:Freeman and Company.

Bassett, S. (2006). Adherence to rehabilitation after anterior cruciate ligament reconstructive surgery. New Zealand Journal of Physiotherapy, 34, 91-92.

Brewer, B. W., Van Raalte, J. L., Cornelius, A. E., Petitpas, A. J., Sklar, J. H., Pohlman, M. H., ... \& Ditmar, T. D. (2003). Protection motivation theory and adherence to sport injury rehabilitation revisited. The Sport Psychologist, 17, 95-103.

Brewer, B. W., Van Raalte, J. L., Petitpas. A. J., Sklar. J. H., Pohlman. M. H., Krushell, R. J., \& Weinstock J. (2000). Preliminary psychometric evaluation of a measure of adherence to clinic-based sport injury rehabilitation. Physical Therapy in Sport, 1, 68-74. doi: 10.1054/ptsp.2000.0019

Cohen, J. (1988). Statistical power analysis for the behavioral sciences (2nd ed.). Hillsdale, NJ: Lawrence Earlbaum Associates.

Cumming, J., \& Ramsey, R. (2008). Imagery interventions in sport. In S. Mellalieu \& S. Hanton (Eds.) Advances in applied sport psychology: A review. London: Routledge. 
Cumming, J., \& Williams, S. E. (2012). The role of imagery in performance. In S. Murphy (Ed), Handbook of Sport and Performance Psychology (p. 213-232). New York, NY: Oxford University Press.

Cupal, D. D., \& Brewer, B. W. (2001). Effects of relaxation and guided imagery on knee strength, reinjury anxiety, and pain following anterior cruciate ligament reconstruction. Rehabilitation Psychology, 46, 28-43. doi:10.1037/0090-5550.46.1.28

Driediger, M., Hall, C., \& Callow, N. (2006). Imagery use by injured athletes: A qualitative analysis. Journal of Sports Sciences, 24, 261-272. doi:10.1080/02640410500128221

Duncan, L. R., Rodgers, W. M., Hall, C. R., \& Wilson, P. M. (2011). Using imagery to enhance three types of exercise self-efficacy among sedentary women. Applied Psychology: Health and Well-being, 3, 107-126. doi:10.1111/j.1758-0854.2010.01043.x

Evans, L., Hare, R., \& Mullen, R. (2006). Imagery use during rehabilitation from injury. Journal of Imagery Research in Sport and Physical Activity. doi:10.2202/1932-0191.1000

Gage, N. A. \& Lewis, J. L. (2013). Analysis of effect for single-case design research. Journal of Applied Sport Psychology, 1, 46-60. doi:10.1080/10413200.2012.660673

Granquist, M. D., Gill, D. L., \& Appaneal, R. N. (2010). Development of a measure of rehabilitation adherence for athletic training. Journal of Sport Rehabilitation, 19, 249-67.

Hare, R., Evans, L., \& Callow, N. (2008). Imagery use during rehabilitation from injury: A case study of an elite athlete. The Sport Psychologist, 22, 405-422.

Hayduck, L. (1996). LISREL Issues, Debates and Strategies. Johns Hopkins University Press, Baltimore. 
Heil, J. (1993). Psychology of sport injury. Champaign, IL: Human Kinetics.

Holden, R. R. \& Jackson, D. N. (1979). Item subtlety and face validity in personality assessment. Journal of Consultancy and Clinical Psychology, 47, 459-468.

Kazdin, A. (2010). Single-case research designs: methods for clinical and applied settings $\left(2^{\text {nd }}\right.$ ed), New York, NY: Oxford University Press.

Kinugasa, T., (2013). The application of single-case research designs to study elite athletes' conditioning: An update. Journal of Applied Sport Psychology, 25, 157-166. doi:10.1080/10413200.2012.709578

Kratochwill, T. R., Hitchcock, J., Horner, R. H., Levin, J. R., Odom, S. L., Rindskopf, D. M. \& Shadish, W. R. (2010). Single-case designs technical documentation. Retrieved from What Works Clearinghouse website: http://ies.ed.gov/ncee/wwc/pdf/wwc_scd.pdf.

Lang, P. J., Kozak, M., Miller, G. A., Levin, D. N., \& McLean, A. (1980). Emotional imagery: Conceptual structure and pattern of somato-visceral response. Psychophysiology, 17, 179192. doi:10.1111/j.1469-8986.1980.tb00133.x

Lox, C. L., Martin Ginis K. A., \& Petruzzello, S. J. (2014). The psychology of exercise: integrating theory and practice. Scottsdale, Arizona: Holcomb Hathaway.

Maddison, R., Prapavessis, H., Clatworthy, M., Hall, C., Foley, L., Harper, T., ... \& Brewer, B. (2011). Guided imagery to improve functional outcomes post-anterior cruciate ligament repair: randomized-controlled pilot trial. Scandinavian Journal of Medicine \& Science in Sports, 22, 816-821. doi:10.1111/j.1600-0838.2011.01325.x 
Mahler, H. I. M., \& Kulik, J. A. (2002). Effects of a videotape information intervention for spouses on spouse distress and patient recovery from surgery. Health Psychology, 21, 427437. doi:10.1037/0278-6133.21.5.427

Martin, G., \& Pear, J. (1996). Behavior modification: What it is and how to do it. Upper Saddle River, NJ: Prentice Hall.

Milne, M., Hall, C., \& Forwell, L. (2005). Self-efficacy, imagery use and adherence to rehabilitation by injured athletes. Journal of Sport Rehabilitation, 14, 150-67.

Munroe-Chandler, K. J., Hall, C. R., Fishburne, G. J., Murphy, L., \& Hall, N. D. (2012). Effects of a cognitive specific imagery intervention on the soccer skill performance of young athletes: Age group comparisons. Psychology of Sport and Exercise, 13, 324-331. doi:10.1016/j.psychsport.2011.12.006

Page, J., \& Thelwell, R. (2013). The value of social validation in single-case methods in sport and exercise psychology. Journal of Applied Sport Psychology, 25, 61-71. doi:10.1080/10413200.2012.663859

Preacher, K. L., \& Hayes, A. F. (2008). Asymptotic and resampling strategies for assessing and comparing indirect effects in multiple mediator models. Behavior Research Methods, 40, 879-891. doi: 10.3758/BRM.40.3.879

Rosnow, R. L., \& Rosenthal, R. (1996). Computing contrasts, effect sizes, and counternulls on other people's published data: General procedures for research consumers. Psychological Methods, 1, 331-340. doi:10.1037/1082-989X.1.4.331 
Rossman, M. (2002). Imagery: the body's natural language for healing. Alternative Therapies in Health and Medicine, 8, 80-89.

Scruggs, T. E., \& Mastropieri, M. A. (1998). Summarizing single-subject research issues and applications. Behavior Modification, 22, 221-242. doi:10.1177/01454455980223001

Scruggs T. E. \& Mastropieri, M. A. (2001). How to summarize single-participant research: ideas and applications. Exceptionality: A Special Education Journal, 9, 227-244. doi:10.1207/S15327035EX0904_5

Sordoni, C., Hall, C., \& Forwell, L. (2002). The use of imagery in athletic injury rehabilitation and its relationship to self-efficacy. Physiotherapy Canada, Summer, 177-185.

Wesch, N., Hall, C., Prapavessis, H., Maddison, R., Bassett, S., Foley, L., ... \& Forwell, L. (2011). Self-efficacy, imagery use, and adherence during injury rehabilitation.

Scandinavian Journal of Medicine \& Science in Sports, 22, 695-703 doi:10.1111/j.16000838.2011.01304.x

West, S. G., Finch, J. F., \& Curran, P. J. (1995). Structural Equation Models with nonnormal variables: Problems and remedies. In Hoyle. P. H (Ed.). Structural Equation Modeling Concepts, Issues, \& Applications. Sage: Thousand Oaks, CA.

Woodgate, J., Brawley, L. R., \& Weston, Z. J. (2005). Maintenance cardiac rehabilitation exercise adherence: Effects of task and self-regulatory self-efficacy. Journal of Applied Social Psychology, 35, 183-222. doi:10.1111/j.1559-1816.2005.tb02099.x 
Table 1.

AIIQ-3 descriptives and zero order correlations between subscales (after item deletion). Alpha coefficients are displayed in bold.

\begin{tabular}{|l|c|c|c|c|c|c|c|c|c|}
\hline Scale & Item means & $\begin{array}{c}\text { Subscale } \\
\text { mean }\end{array}$ & $\begin{array}{c}\text { Subscale } \\
\text { SD }\end{array}$ & Skewness & Kurtosis & 1 & 2 & 3 & 4 \\
\hline 1. Cognitive & $3.64-4.62$ & 4.18 & 1.98 & -0.93 & 0.16 & $\mathbf{. 8 6}$ & & & \\
2. Motivational & $4.70-5.59$ & 5.17 & 1.57 & -0.34 & -.91 & .72 & $\mathbf{. 8 2}$ & & \\
3. Healing & $3.25-4.15$ & 3.73 & 1.72 & 0.97 & -1.32 & .64 & .65 & $\mathbf{. 9 1}$ & \\
4. Pain & $3.60-4.87$ & 4.29 & 1.72 & -0.31 & -0.92 & .79 & .87 & .79 & $\mathbf{. 8 2}$ \\
\hline
\end{tabular}


Table 2.

Factor Loadings for the items on the Athletic Injury Imagery Questionnaire

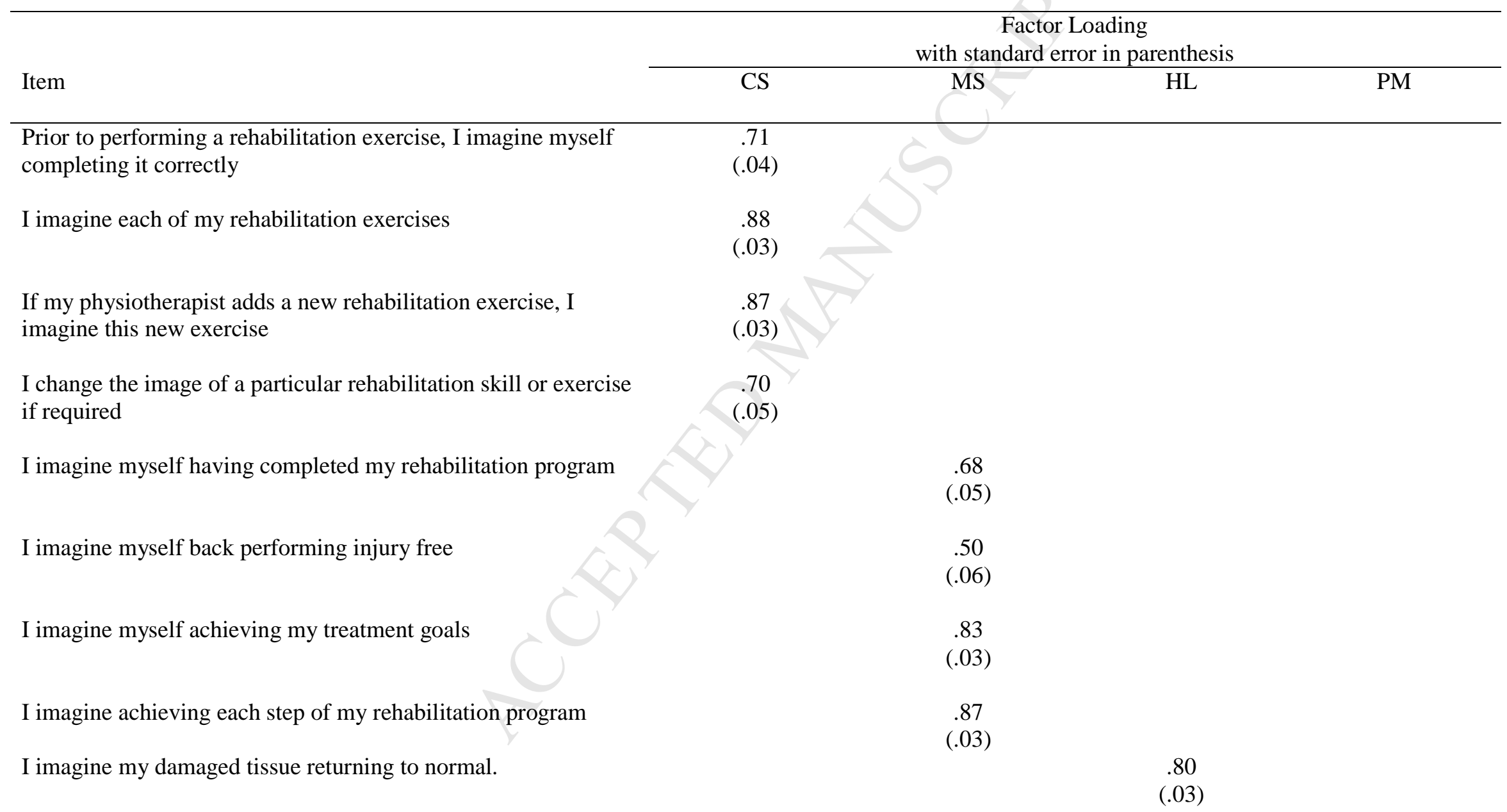


I imagine my bone or tissue growing as my injury mends

I imagine the physiological changes my body is undergoing

I imagine myself working through the pain when rehabilitating

Note. Factor loadings can range from 0-1; all item loading values in the analysis exceed the minimum criterion value of .45

$\mathrm{CS}=$ Cognitive Specific; $\mathrm{MS}=$ Motivational Specific $; \mathrm{HL}=$ Healing; $\mathrm{PM}=$ Pain Management 
Table 3.

Task-efficacy results

\begin{tabular}{|c|c|c|c|c|c|}
\hline & \multicolumn{2}{|c|}{ Level and Variability } & \multicolumn{2}{|c|}{ Trend } & Immediacy of \\
\hline Part & Baseline & $\begin{array}{c}\text { Post- } \\
\text { intervention }\end{array}$ & Baseline & $\begin{array}{c}\text { Post- } \\
\text { intervention }\end{array}$ & \\
\hline 1 & $\begin{array}{c}0.00 \\
(0.00)\end{array}$ & $\begin{array}{c}83.67 \\
(12.88)\end{array}$ & Stable & Increasing & Delayed \\
\hline 2 & $\begin{array}{l}81.67 \\
(5.77)\end{array}$ & $\begin{array}{l}90.00 \\
(3.56)\end{array}$ & Stable & Stable & Delayed \\
\hline 3 & $\begin{array}{l}86.67 \\
(0.00)\end{array}$ & $\begin{array}{l}89.33 \\
(4.10)\end{array}$ & Stable & Stable & Immediate \\
\hline 4 & $\begin{array}{l}85.00 \\
(1.83)\end{array}$ & $\begin{array}{l}100.00 \\
(0.00)\end{array}$ & Stable & Stable & Immediate \\
\hline 5 & $\begin{array}{c}59.52 \\
(10.57)\end{array}$ & $\begin{array}{l}92.00 \\
(7.67)\end{array}$ & Decreasing & Increasing & Immediate \\
\hline 6 & 81.67 & $\mathrm{n} / \mathrm{a}$ & $\mathrm{n} / \mathrm{a}$ & $\mathrm{n} / \mathrm{a}$ & $\mathrm{n} / \mathrm{a}$ \\
\hline
\end{tabular}


Table 4.

Coping-efficacy results

\begin{tabular}{lccccc}
\hline & \multicolumn{2}{l}{ Level and Variability } & \multicolumn{2}{c}{ Trend } & $\begin{array}{c}\text { Immediacy of } \\
\text { effect }\end{array}$ \\
\hline Part & Baseline & $\begin{array}{c}\text { Post- } \\
\text { intervention }\end{array}$ & Baseline & $\begin{array}{c}\text { Post- } \\
\text { intervention }\end{array}$ & \\
\hline 1 & 0.00 & 86.25 & Stable & Increasing & Immediate \\
2 & $(0.00)$ & $(16.75)$ & & Stable & $\mathrm{n} / \mathrm{a}$ \\
& 90.83 & 99.06 & Decreasing & Stable & $\mathrm{n} / \mathrm{a}$ \\
3 & $(3.91)$ & $(1.29)$ & & & \\
& 87.50 & 87.50 & Stable & Immediate \\
& $(0.00)$ & $(2.89)$ & Stable & Stable & \\
5 & 85.00 & 95.00 & & & Delayed \\
& $(3.16)$ & $(2.74)$ & & Increasing & n/a \\
\hline
\end{tabular}


Figure 1.

Graphed task efficacy data for Participants 1, 2, 3, 4, 5, and 6.
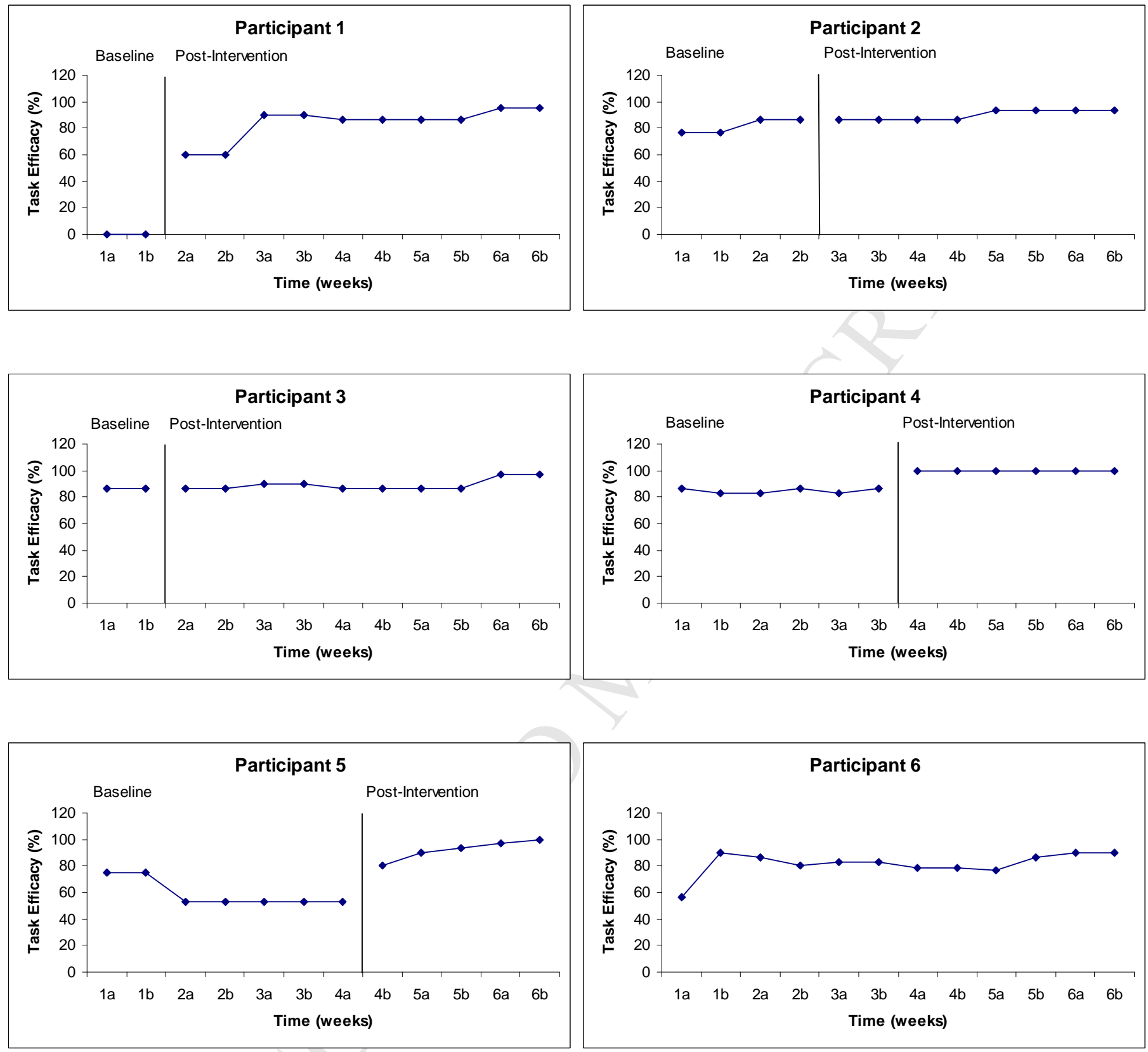

Note. Participant 6 did not receive the intervention. The solid vertical line on each graph indicates the point at which the intervention was implemented for that participant 
Figure 2.

Graphed coping efficacy data for Participants 1, 2, 3, 4, 5, and 6.
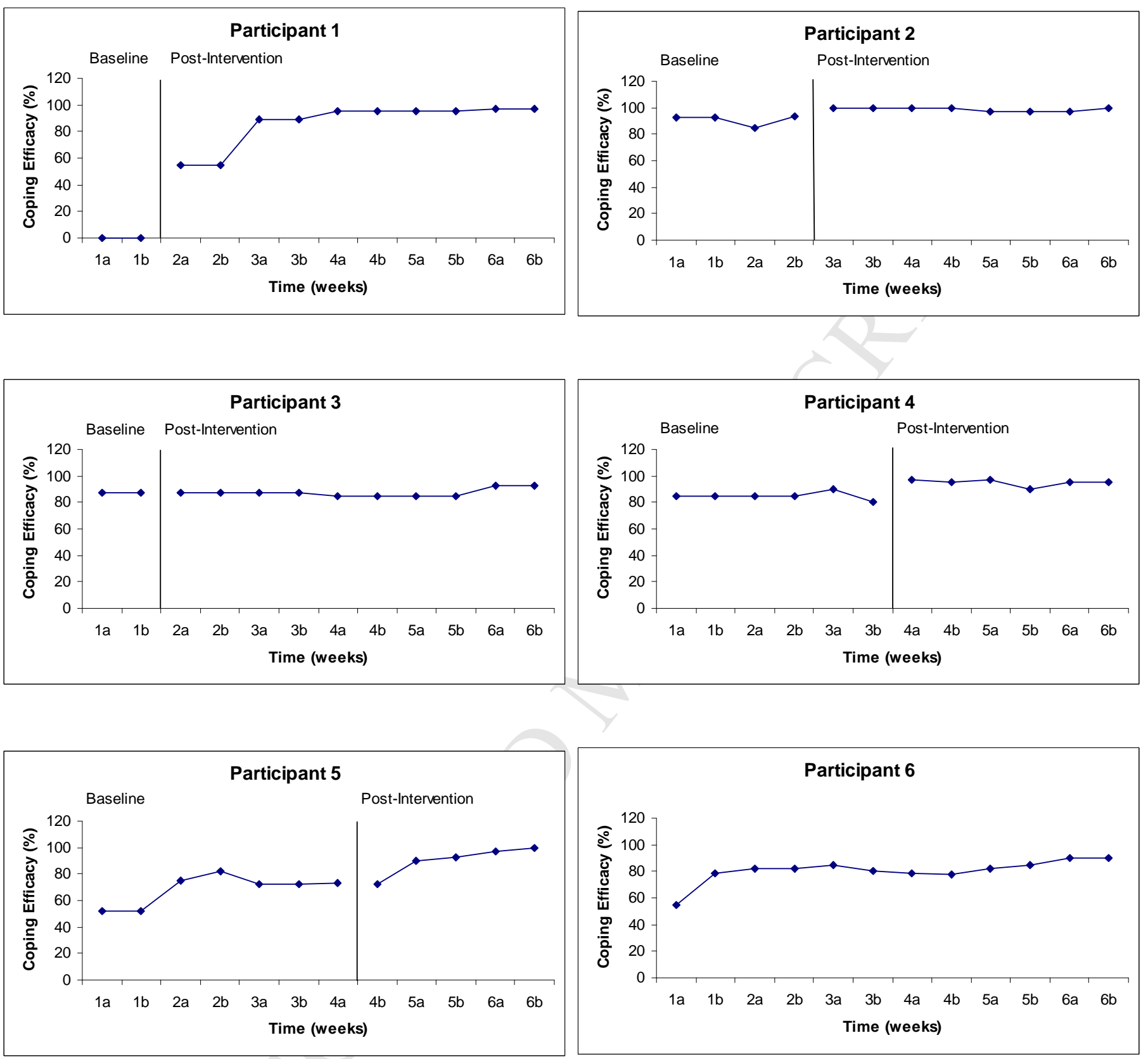

Note. Participant 6 did not receive the intervention. The solid vertical line on each graph indicates the point at which the intervention was implemented for that participant. 
Development of pain management scale for the Athletic Injury Imagery Questionnaire-2

Evidence for the factorial validity of the adapted questionnaire provided

Imagery intervention administered to injured athletes prior to commencing physiotherapy

Positive effects of imagery intervention on task and coping efficacy revealed

Future measurement and imagery-intervention related research proposed 